\title{
Recherche de gaz de substitution au bromure de méthyle pour la désinsectisation des biens patrimoniaux dans les monuments historiques
}

\section{Katia Baslé}

\section{(2) OpenEdition}

\section{Journals}

Édition électronique

URL : http://journals.openedition.org/ocim/986

DOI : $10.4000 /$ ocim.986

ISSN : 2108-646X

Éditeur

OCIM

Édition imprimée

Date de publication : 1 novembre 2011

Pagination : 26-27

ISSN : 0994-1908

\section{Référence électronique}

Katia Baslé, «Recherche de gaz de substitution au bromure de méthyle pour la désinsectisation des biens patrimoniaux dans les monuments historiques », La Lettre de I'OCIM [En ligne], 138 | 2011, mis en ligne le 01 novembre 2013, consulté le 02 mai 2019. URL : http://journals.openedition.org/ocim/986 DOI : 10.4000/ocim.986

Ce document a été généré automatiquement le 2 mai 2019.

Tous droits réservés 


\title{
Recherche de gaz de substitution au bromure de méthyle pour la désinsectisation des biens patrimoniaux dans les monuments historiques
}

\author{
Katia Baslé
}

1 L'étude détaillée a fait l'objet d'une communication au colloque «Cultural Heritage Pests » qui s'est tenu du 7 au 9 juin 2011 au Centro per la Protezione dei Beni Culturali à l'université de Piacenza en Italie; elle est à paraître prochainement dans le Journal of Entomological and Acarological Reasearch.

2 Le programme de recherche a été mené en partenariat avec :

- le Centre interrégional de Conservation et Restauration du Patrimoine (CICRP), Marseille par Katia Baslé (chef de travaux d'art) ;

- l'Institut de Recherche sur les Archéomatériaux, UMR CNRS 5060, Centre de Recherche en Physique appliquée à l'Archéologie (CRPAA), université Bordeaux 3, par le docteur Floréal Daniel (ingénieur de recherche), et le docteur Aurélie Mounier ;

- le docteur Luc Robbiola, Laboratoire TRACES - UMR CNRS 5608 ;

- Inocence Queixalos (conservateur-restaurateur) ;

- le Laboratoire national des Denrées stockées (LNDS), Villenave-d'Orno, par Patrick Ducom et Jérôme Fritsch.

3 Tous les secteurs concernés par la protection du patrimoine sont touchés par les problèmes d'infestation. Les bâtiments historiques et leurs collections y sont confrontés de manière plus problématique en raison de la difficulté d'intervention dans les lieux in situ et la difficulté liée au fait que les œuvres, bien souvent, sont intransportables et non démontables. L'environnement de ces lieux est propice au développement des insectes et moisissures : les Monuments historiques, caractérisés par une diversité des œuvres et des matériaux, les bibliothèques et les archives, les musées (réserves et collections). Il est fréquent que la désinfection d'objets mobiliers dans le cadre de Monuments historiques 
agisse sur des éléments de décor comme les enduits peints, stucs dorés... Les peintures murales, en particulier, comportent des pigments et des éléments métalliques sensibles (dorures à la mixtion, à la détrempe, à la coquille, bronzine...). Il était important d'étu dier, sur des échantillons choisis, l'effet des traitements de fumigation sur ces éléments de notre patrimoine.

Souvent, les attaques sur les matériaux, en particulier le bois, nécessitent des traitements chimiques de désinsectisation. Jusqu'à présent, un gaz était utilisé, le bromure de méthyle, mais depuis le premier janvier 2007, et suite à la directive 2006/140/CE du 20 décembre 2006 il a été interdit et définitivement abandonné pour les usages biocides au profit du fluorure de sulfuryle. En 2008, dans le domaine patrimonial s'est posée la question de l'utilisation de ce fumigant en tant que gaz de substitution ainsi que celles du phosphure d'hydrogène (PH3) et du disulfure de diméthyle (DMDS). Le fluorure de sulfuryle et le phosphure d'hydrogène sont des gaz homologués et validés en usage biocide et phytosanitaire, le DMDS a été homologué en juin 2010, aux États-Unis, en tant que désinfectant pour les sols en usage phytosanitaire. Il nous est apparu intéressant de l'intégrer, ainsi que l'éthane dinitrile (C2N2) afin d'évaluer les interactions physicochimiques avec les matériaux du patrimoine culturel de tous les candidats possibles au remplacement du bromure de méthyle. Afin d'observer les effets de ces gaz dans le temps, les échantillons ont été altérés artificiellement, selon un vieillissement hygrothermique et un vieillissement à la lumière, et comparés aux échantillons de référence ayant subi les mêmes vieillissements.

5 L'objectif d'une telle étude serait de pouvoir trancher en faveur de l'un ou l'autre de ces gaz. Cependant chacun possède ses avantages et ses limites, et le choix doit répondre à un compromis entre tous les paramètres, que ce soit la mise en œuvre, le coût, les altérations des matériaux... :

- l'étude menée sur les bois dorés montre que le DMDS, qui n'est pas encore homologué, pourrait être satisfaisant, n'était son odeur d'ail très persistante, et ce jaunissement du vernis dammar à la lumière. Il convient cependant d'être prudent avec ce dernier cas, qui demanderait confirmation. Cependant, si ces effets se confirmaient, ils conduiraient sans doute à un abandon de ce gaz pour cet usage. De plus, si le DMDS et le cyanogène ont des effets moins corrosifs que la Phosphine, ils conduisent cependant sur tous les métaux étudiés à une altération, plus prononcée pour le cuivre et le plomb, et induisent une instabilité des composés au cours du vieillissement. Ils ne sont donc pas à recommander ;

- le fluorure de sulfuryle est également satisfaisant, les impuretés de ces substances pouvant être contrôlées. Les études menées par le Getty Conservation Institute étaient favorables à l'utilisation du fluorure de sulfuryle sur des objets du patrimoine culturel. De nombreuses églises ont été traitées en Allemagne notamment, sans qu'aucun effet notoire n'ait été relevé, et récemment en France, la première fumigation de monument historique, l'église de Hauteluce (73), a été réalisée en 2007 ;

- le phosphure d'hydrogène n'est pas du tout satisfaisant. En effet, ses effets irréversibles sur les alliages cuivreux l'éliminent complètement, malgré une certaine facilité de mise en œuvre. Sur les enduits peints, c'est le gaz qui provoque le plus d'altérations de la couleur immédiatement après le traitement, en particulier sur les dorures à l'or. Il n'est absolument pas recommandé pour la désinsectisation d'un local patrimonial en présence d'objets métalliques en argent, en cuivre (ou alliage contenant du cuivre), en étain, en plomb ou en fer. 
- les résultats et observations montrent que l'emploi du C2N2 comme moyen de désinfection semble compromis et non adapté à ce type de matériaux.

- le fluorure de sulfuryle (Vikane) est le traitement le plus convenable en tant que gaz de substitution au bromure de méthyle, les métaux dans leur ensemble présentant un meilleur comportement. Il semblerait donc être un candidat idéal en substitution du bromure de méthyle. Son coût reste cependant très élevé, et au moins le double de substance active est nécessaire pour la même efficacité.

\section{BIBLIOGRAPHIE}

Baker, M.-T., Burgess, H.-D., Binnie, N.-E. Derrick, M.-R. et Druzik, J.-R. Laboratory investigation of the fumigant vikane, 9th Triennial meeting, ICOM, Dresde, 26-31 août 1990, Los Angeles, pp. 804-811.

Bernard, M.-C. et Joiret, S.Understanding corrosion of ancient metals for the conservation of cultural heritage, Electrochimica Acta, ${ }^{\circ}$ 54, 22, 2009, pp. 5199-5205.

Bertholon, R. Corrosion du cuivre et de ses alliages lors d'un traitement de désinfestation par fumigation à partir de phosphine, Actes de la 7e rencontre annuelle du groupe de travail de la section métal, ICOM-SFIIC, Draguignan, 23 avril 1993, pp. 1-13.

Binker, G. Report on the first fumigation of a church in Europe using sulfuryl fluoride, Proceedings of the 1 st international conference on insect pests in the urban environment, Cambridge, 30 juin-3 juillet 1993 , pp. 51-55.

Bouchard, M. et Smith, D. Catalogue of 45 reference Raman spectra of minerals concerning research, Art history or archaeology, especially on corroded metals and coloured glass, Spectrochimica Acta, n 59, 2003, pp. 2247-2266.

Costa,V. et Urban, F. Lead and alloys: metallurgy, deterioration and conservation, Reviews in Conservation, $\mathrm{n}^{\circ}$ 6, 2005, pp. 48-62.

Costa, V. Impact of environmental conditions on metallic artefacts from the treasure rooms of Reims Cathedral, Heritage, Weathering and Conservation. Fort : Alvarez de Buergo, Gomez-Heras \& Vasquez-Calvo (eds), vol. 1, 2006, pp. 453-456.

Delfane, B. Caractérisation électroanalytique et physico-chimique des produits d'altération de matériaux métalliques culturels : cas du cuivre, rapport de stage d'ingénieur (sous la direction de V. Costa), ENSCP Paris, juin 2006, $42 \mathrm{p}$.

Derrick, M.-R., Burgess, H.-D, Baker,M.-T. et Binnie, N.-E. Sulfuryl fluoride (vikane): a review of its use as a fumigant, Journal of the American Institute for Conservation, vol. 29, n 1, 1990, pp. 77-90.

Ducom, P. Lutte contre les insectes par gazage : aspect réglementaire, types de gaz, contrôles d'efficacité, in Biodétérioration et désinfection des collections d'archives et de bibliothèques, Actes des 2es journées sur la conservation préventive, Arles, 18-19 novembre 1996, pp. 112-126.

Dupont, D. et Steen, D. Colorimétrie, Mesure des couleurs de surface, Techniques de l'ingénieur, article $\mathrm{n}^{\circ} \mathrm{R} 6442,2006$. 
Feller, R.-L. Accelerated aging. Photochemical and thermal aspects, The Getty Conservation Institute, Los Angeles, 1994.

Hayez, V. Use of micro-Raman spectroscopy for the study of the atmospheric corrosion of copper alloys of cultural heritage, Thèse de doctorat, Vrije Universität, Brussels, Faculteit Ingenieurswetenschappen, departement Metallurgie, Elektrochemie \& Materialenkennis, 2006.

Koestler, R.-J., Parreira, E., Santoro, E.-D. et Noble, P. Visual effects of selected biocides on easel painting materials, Studies in conservation, $\mathrm{n}^{\circ} 38,1993$, pp. 265-273.

Masschelein-Kleiner, L., Heylen, J. et Tricot-Marckx, F. Contribution à l'analyse des liants, adhésifs et vernis anciens, Studies in Conservation, n 13, 1968, pp. 105-121.

Neff, D., Bellot-Gurlet, L., Dillmann, P., Reguer, S. et Legrand, L. Raman imaging of ancient rust scales on archaeological iron artefacts for long-term atmospheric corrosion mechanisms study, Journal of Raman spectroscopy, $\mathrm{n}^{\circ} 37,2006, \mathrm{pp} .1228-1237$.

Perrault, G. Dorure et polychromie sur bois. Dijon : Éditions Faton, 1992.

Regert, M., Guerra, M.F. et Reiche, I. Physico-chimie des matériaux du patrimoine culturel, partie 1, Techniques de l'ingénieur, article n P 3780, 2006.

Salvant, J. Évaluation électrochimique de l'impact environnemental sur la conservation des objets métalliques du patrimoine, rapport de stage d'ingénieur, (sous la direction de V. Costa), ENSCP Paris, juin 2006, $28 \mathrm{p}$.

$\mathrm{Su}$, N.-Y. Efficacy of Sulfuryl Fluoride against selected insect pest of museums, Proceedings of the 2nd International Conference on Biodeterioration of cultural property, Yokohama, 5-8 octobre 1992, pp. 434-439.

Vega, E. Altération des objets ferreux archéologiques du site de Glinet (Seine-Maritime, France, XVIe siècle) : caractérisation des produits de corrosion et étude des mécanismes, thèse de doctorat, université de Technologie de Belfort Montbéliard, 2004, pp. 47-72.

\section{AUTEUR}

\section{KATIA BASLÉ}

Katia Baslé est chef des travaux d'art, coordination préventive au Centre interrégional de Conservation et Restauration du Patrimoine (CICRP) de Marseille. katia.basle@cicrp.fr 